\title{
From product-oriented to process-oriented: Emerging new engineering paradigm
}

\author{
S FUKUDA \\ Tokyo Metropolitan Institute of Technology, 6-6. Asahigaoka, Hino, Tokyo 191, Japan

\begin{abstract}
The conventional approach has put emphasis upon reducing labour, and increasing efficiency and uniformity. But this holds good only in the mass production paradigm. As we are entering the age of small production with far wider varieties, these principles of yesterday should be re-examined. Labour itself sometimes gives satusfaction if it involves the joy of creation, and longer time does not necessarily mean just patience, it sometimes turns into expectation if greater joy will be provided later. Too much fitness for the purpose sometimes means lack of robustness and adaptability to the quickly and widely changing environment. Thus, we should reconsider the items we thought important in the mass production age to aptly cope with the diversification of each customer's requirements.
\end{abstract}

Keywords. Product diversification; paradigm shift; process-oriented; human-oriented.

It may be said that the conventional product development has been too much product-oriented. The value of a product has been evaluated only on the basis of performance of the final product, and most of the processes in product realization have been regarded only as cost increasing factors. And efficiency, in terms of less time and cost, has been emphasized all the time.

This may be adequate in the traditional mass production age when people desired to obtain a product in shorter time and at a lesser price. But now we are living in abundance of products, and people have diverse requirements.

In the conventional product development, products have been realized by undergoing processing sequentially (figure 1), where only necessary pieces of information are passed on to the next stage and decisions are based on them alone.

Thus, as we go downstream, the constraints become increasingly severe and the search space narrow (figure 2). Consequently, a production engineer was considered competent if he or she could come up with best solution under these constraints.

In the product development, the number of combinations increases as we go downstream. The more the requirements of customers, the more the number of combinations increases and severer the constraints become (figure 2). So the reduction of the burden of engineers at downstream stages becomes really pressing these days. Thus, increasing complexities and diversification of products makes this approach practically impossible.

It must be noted that the role of a material engineer in this sense is not different from that of a production engineer as they both have to make decisions based upon the specifications a design engineer provides. That is, if we consider a structure, geometrical considerations will probably be given the first priority and then suitable materials will be sought next. This has been the situation up to now.

But if we consider newer fields like space, micromachines, etc this situation is rapidly changing. For instance in space it is of utmost importance whether we can satisfy the requirements with the minimum weight, and therefore space engineers try to integrate structural, material, control and other aspects and consider these aspects concurrently. 


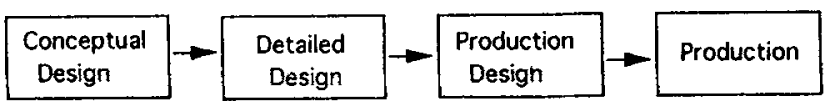

Figure 1. Flow of conventional product development.

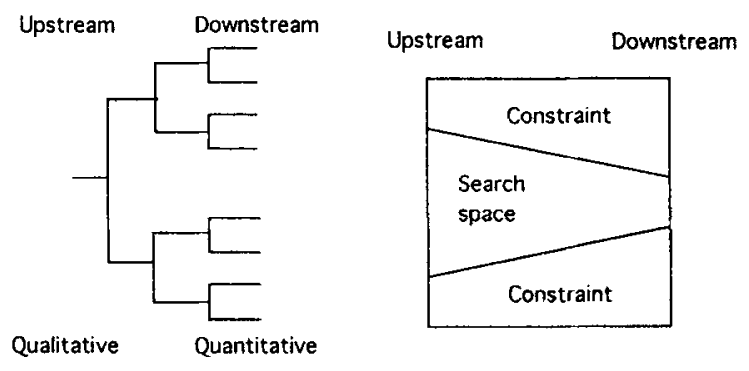

Figure 2. Conventional product development.

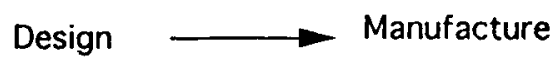

(a) Conventional products

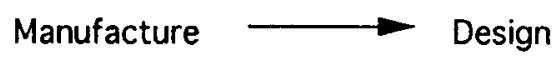

(b) Micromachines

Figure 3. Micromachine development.

In the case of a micromachine aspects of production, that is, whether we can manufacture or not comes first. Other example is in the use of mechatronics products in our daily life, which require mechanical and electrical considerations at the same time in their developments. This is opposed to the conventional approach (figure 3 ), wherein we started from design and then went on to manufacture.

To cope with these fieids requiring multiple technologies, we have to integrate all the processes and to consider them concurrently. Then, we can make the constraints, hence the burden, equally distributed all through the processes (figure 4).

To achieve such concurrency in product realization, we have to set up a common basis among engineers in different process domains. Then the one way flow in the traditional product realization will be changed so that information can be passed on either way. To attain such a goal we have to make each process visible to engineers in other domains so that they can understand the idea and share a common perspective.

In the mass production age the task of finding a goal was comparatively easy, since the conditions did not change from product to product appreciably. But in the age of diversification this task becomes crucially important, since a goal changes from product to product and in consequence a wider knowledge and experience are required for the engineers in different domains to collaborate. 


\section{Upstream Downstream}

\begin{tabular}{|c|}
\hline Constraint \\
\hline $\begin{array}{l}\text { Search } \\
\text { space }\end{array}$ \\
\hline Constraint \\
\hline
\end{tabular}

Figure 4. Concurrent product development.

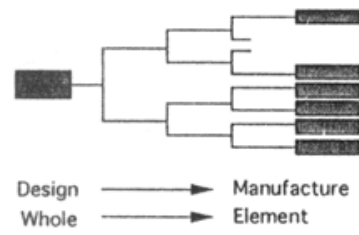

(a) Conventional design

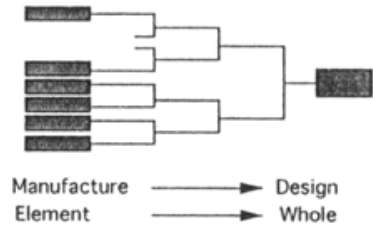

(b) Reverse design

Figure 5. Change in product development.

In the mass production age products were developed in a hierarchical system where information was passed down from top to bottom. The roles and responsibilities were distributed among people by the instructions from the top. And the task of finding a goal was not necessarily needed at the middle class and under because a goal did not change appreciably from case to case.

But in an age of diversification we have to turn the system into a network so that we can really utilize the knowledge and experience of others to the full extent. In a network system information is passed both ways and in a very interactive manner. Therefore, goal finding will be the most important task and building up the common basis will be a prerequisite for sharing the view. Thus, we have to make our technologies visible and understandable to others in different domains.

How can we make our technologies understandable to others? Some of the ways we can introduce are, for example, the Taguchi Method which separates factors into independent ones using a statistical approach. Prof. N P Suh at MIT also stresses the importance of separating the design factors into functionally independent ones. These methods will serve to make our technologies understandable to others or to make it easier to reorganize them for another application. Of course, other approaches are also possible, but what should be stressed here is that we make our technologies transparent and easy for reorganization.

In fact this is very consistent with the new quality assurance concept such as ISO 9000 , where they are talking about whether a 'process' is capable of producing a good quality product or not. What they are questioning is not only the quality as a final product, but whether the processes really satisfy the requirements for producing good quality products. This is completely different from the conventional quality assurance approach. 
The conventional approach assured us that the quality is good, but did not assure that the same kind of quality can be obtained for other kinds of products as well. Thus, it was valid when the same or quite similar kinds of products were produced. But the quickly diversifying requirements make this kind of approach unrealistic, since the conditions in design and production vary largely from product to product so that no matter how good quality one product is, we can not be sure of the qualities of other kinds of products. In such a situation, we will not be assured unless the procedures of securing quality become visible to us. Hence, making technologies transparent becomes a very important issue today.

If we can successfully solve the problem of securing concurrency and transparency of technologies, then we will not only more aptly deal with diversifying requirements, but we will also be more creative in product development. In fact the ideas of intelligent materials or intelligent structures are some good examples. In the former the material provides the functions of sensors, actuators, etc in addition to the ordinary ones concurrently.

The above discussion on collaboration is made mainly with engineers in mind. But it should also be stressed that if we really can make our technologies more visible and more accessible to others, we may also be successful in letting the customers themselves get involved in the product development. It, however, must be admitted that the product developments today are much too complicated and diversified for a layman to interfere in. Still, if the technologies can be made more transparent, we may be able to have the customer involvement in the product development processes. At least it will be possible for them to show their preferences more often in a more interactive manner during the realization process. This would provide them with more attractiveness or satisfaction when they consider buying a product.

Let us consider, for example, the case of an automobile. Today every automobile company tries to deliver a car to their customers in as much short time as possible. But if the customers can participate in the processes, then they might be more patient and wait for a longer time and even accept a higher price if their preferences can be taken into consideration and realized in a product. Thus, the processes themselves will yield values.

It should also be added that if such process-oriented approach can be realized, artistic value can be easily added to the processes, as, today people are getting more and more interested in art than before. In fact, the fusion of art and technology will be the next challenge facing us.

Turning back to technological problems, let us consider, for example, the problem of tolerances. Up to now we have been determining the amount or degree of tolerances independently at each stage. But if we can consider the problem of tolerance all through the product realization processes or further through its life cycle, then we will be able to determine more reasonable values.

Further, if the customers can be involved in the processes, they might be satisfied with lower grade materials, for example, with less pressing quality, saying that their cars are more artistic than other ones!

We have been producing products in a 'product out' manner, i.e. we have been producing on the assumption that the customers desire such kinds of products. But as the customers have been cut off from the product realization processes, we do not really know what the customers desire.

If we can move towards more process-oriented direction, we will be able to realize a true 'market in' product development and a new kind of material science will emerge, where multiple technologies will be fused into one. 
Finally let us consider the problem of non-destructive inspection. The conventional NDI assured us that the design requirements are fully satisfied. But the diversification calls for another kind of NDI where design, material, and other aspects are considered concurrently as exemplified by an intelligent material. The conventional NDI verified at the last stage that the product fully complies with the design requirements. To cope with the diversification, more positive and frequent feed back and interaction with design is needed. Further, more attention should be paid to the operational aspects, since these data will serve a great deal when we wish to develop a new kind of intelligent material.

To realize such an intelligent material, concurrent product development system will be necessary, where all the required functions are considered and developed at the same time in a concurrent and integrated manner. The NDI data will provide a basic foundation for such procedures. But it should be pointed out that the present day NDI are too much technique-specific. There are X-ray specialists, UT specialists, etc in each domain of technique, but very few experts who can diagnose as a whole. We sometimes forget how important a visual inspection is. It is very rough and as far as accuracy is concerned, it may not be worth the name of NDI. But the importance of such overall inspections or such sensory feelings that something is wrong somewhere could not be overemphasized if we consider designing an intelligent material or an intelligent structure. The integration of many data obtained by different techniques is a pressing matter in this respect.

The product development approach we have discussed may be called a 'reverse design', since in the conventional design, procedures start with designing the whole and then break them into elements and finally assemble them together into a product. But in this 'reverse design', procedures start with designing the elements and integrate them into the final product by paying attention to technological concurrency (figure 5).

Japanese products are known to have good quality. But I would say our approach is very fragile. We, Japanese can work together very well and produce a good quality product if the goal is clear. But in this age of diversification, the most important task is to determine what we should do. Japanese work hard to secure good quality without paying much attention to the customer's requirements. Or in other words, Japanese do their best to attain the absolute level of quality which they themselves set as a target. But Japanese are very poor in complying with the customer's requirements, for instance if a customer desires downgrading, it would be quite difficult for Japanese to respond to his or her request. However, if Japanese introduce more process oriented approach, the whole situation will be changed.

In conclusion I would like to say that we should move towards the process-oriented product development. Then we will be more flexible, versatile and robust in coping with the diversifying requirements.

\section{References}

Fukuda S 1993 Concurrent engineering (Tokyo: Baifukan) (in Japanese)

Fukuda S, Logcher R and Sriram D 1991 Computer-aided cooperative product development (Berlin: Heidelberg, Springer-Verlag) 